\title{
Virtuelle Ausstellungen aus der WLB
}

Im Oktober 2019 präsentierte die Deutsche Digitale Bibliothek (DDB) den von ihr bereitgestellten Service DDBstudio. ${ }^{2}$ Mit diesem digitalen Arbeitswerkzeug, das auf der vom Roy Rosenzweig Center for History und New Media ${ }^{3}$ entwickelten OpenSource-Software Omeka basiert, können deutsche Kultur- und Wissenseinrichtungen, die sich bei der DDB zuvor haben registrieren lassen, browserbasiert virtuelle Ausstellungen einrichten und gestalten. Der Ausstellungsservice der DDB folgt dabei ihrem generellen Auftrag:

„Ziel der Deutschen Digitalen Bibliothek (DDB) ist es, jedem über das Internet freien Zugang zum kulturellen und wissenschaftlichen Erbe Deutschlands zu eröffnen, also zu Millionen von Büchern, Archivalien, Bildern, Skulpturen, Musikstücken und anderen Tondokumenten, Filmen und Noten. Als zentrales nationales Portal soll die DDB perspektivisch die digitalen Angebote aller deutschen Kultur- und Wissenschaftseinrichtungen miteinander vernetzen. Mit der DDB soll Deutschland seine Anschluss- und Wettbewerbsfähigkeit in Wissenschaft, Forschung und Bildung sichern, aber auch sein einzigartiges kulturelles Erbe und Wissen für alle komfortabel über einen zentralen Anlaufpunkt zugänglich machen. Durch die zentrale Zugänglichkeit, indem an jedem PC-Arbeitsplatz mit Internetanschluss unabhängig von Ort und Zeit Zugang zur gesamten erforderlichen Information geschaffen wird, werden die Recherchemöglichkeiten in Forschung, Lehre und Wirtschaft grundlegend verbessert." 4

Dass dies ein andauernder Prozess ist, versteht sich dabei fast von selbst. Aber gegen Ende Februar 2021 verzeichnete die DDB, die seit November 2012 in einer Beta-Version und seit dem 31.3.2014 in Vollversion online ist, bereits über 36 Millionen Objekte, von denen knapp 12 Millionen mit einem Digitalisat verknüpft sind. ${ }^{5}$ Seit dem Launching des Ausstellungstools DDBstudio im Oktober 2019 wurden zudem bereits 68 virtuelle Ausstellungen von 40 Einrichtungen kuratiert und freigeschaltet. ${ }^{6}$ Das jeweils aktualisierte Gesamtverzeichnis findet sich unter https://www.deutsche-digitale-bibliothek. de/content/journal/ausstellungen.
Als die Entscheidung der WLB zur Kuratierung Virtueller Ausstellungen über DDBstudio kurz nach der Präsentation des digitalen Tools im Oktober 2019 fiel und die zuständigen Kolleg:innen sich entschlossen, die Möglichkeiten der Software zunächst mit der virtuellen Übernahme, Erweiterung und Transformation zweier Kabinettausstellungen auszuprobieren, die im Jahr 2018 in der Interimsausstellungsfläche „Zwischen Räumen“ in Stuttgart gezeigt worden waren (dazu unten mehr), waren die Folgen der Corona-Pandemie auch für den deutschen Wissenschafts- und Kulturbetrieb noch nicht absehbar. Der Testlauf für zukünftige zusätzliche Angebote stellte sich während der Wochen der präventionsbedingten Schließung der Württembergischen Landesbibliothek dann bereits als Glücksfall dar. Ausstellungsinhalte waren in Zeiten der SchlieBung des Hauses auch ortsunabhängig zugänglich. Während für die große Ausstellung „Aufbrüche - Abbrüche. 250 Jahre Friedrich Hölderlin“, die über lange Wochen leider nicht vor Ort zugänglich war, eine virtuelle Fassung über DDBstudio erst noch entsteht und im Laufe des Jahres 2021 zugänglich werden soll (auch dazu unten mehr), steht ein Entschluss der WLB bereits fest: In Verbindung mit den Ausstellungen vor Ort sollen zukünftig virtuelle Fassungen möglichst immer mitkuratiert und bereitgestellt werden.

Maik Bozza

\section{8:}

\section{Zwischen Weltkrieg und Revolution}

Das letzte Jahr des Ersten Weltkriegs war für die Deutschen gekennzeichnet von Hoffnungen, Enttäuschungen, Niederlage und Revolution. Sah es zu Beginn des Jahres so aus, als könnte Deutschland den Krieg doch noch gewinnen, zeichnete sich im Laufe des Sommers die drohende Niederlage ab.

1) Vgl. zum Folgenden die Pressemitteilung der DDB vom 8.10.2019, vgl. http://cms.deutsche-digitale-bibliothek.de/sites/default/files/media/document/2019-10/PM_DDBstudio_Launch_191008.pdf bzw. https://www.deutsche-digitale-bibliothek.de/content/ueber-uns.

2) Vgl. https://pro.deutsche-digitale-bibliothek.de/ddbstudio.

3) https://rrchnm.org

4) https://www.deutsche-digitale-bibliothek.de/content/ueber-uns.

5) Genaue Angaben: „36.131.367 Objekte, davon 11.949.153 mit Digitalisat", abgerufen unter https://www.deutsche-digitale-bibliothek.de am 25.02.2021.

6) Für die Informationen danke ich Lidia Westermann von der DDB. 


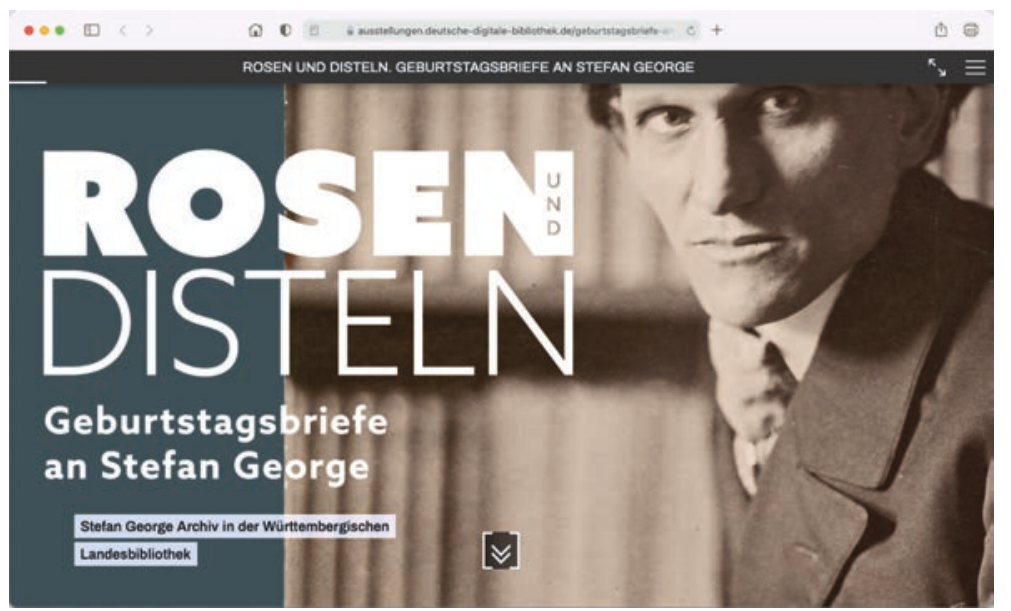

Vereinnahmungswille, gelegentlich auch nur der unbedingte Wunsch nach einem Autogramm ... In jedem Fall passt Georges wohl berühmtester Vers, "Komm in den totgesagten Park und schau:", ${ }^{10}$ als Einladung auch zur digitalen Ausstellung:

https://ausstellungen.deutsche-digitale-bibliothek. de/geburtstagsbriefe-an-george/

Maik Bozza

\section{Aufbrüche - Abbrüche. 250 Jahre Friedrich Hölderlin}

Die Württembergische Landesbibliothek präsentiert noch bis zum 30. April 2021 die Ausstellung "Aufbrüche - Abbrüche. 250 Jahre Friedrich Hölderlin“ auf der Grundlage der umfangreichen Sammlungen des Hölderlin-Archivs. Sie ist als breite Archivausstellung konzipiert, richtet ihren Fokus also bewusst auf mehrere große Themenfelder. Hölderlins Stuttgarter Aufenthalt gehört ebenso dazu wie seine Frankreichreise sowie seine große Liebe zu Susette Gontard. Diese biografischen Themengebiete werden ergänzt durch verschiedene Bereiche seiner komplexen Rezeptionsgeschichte sowie dem Gebiet "Hölderlin digital“. Entsprechend vielschichtig stellt sich die Auswahl der insgesamt 200 Exponate dar, die sich auf 14 Wand-, 13 Tisch- und 2 Stehvitrinen verteilen und in das neue Ausstellungsmobiliar des Neubaus integriert sind. Die Wirkung der Ausstellung resultiert aus dieser räumlichen Komponente in Verbindung mit einer besonderen, individuellen Gestaltung auf der Basis der eigens für das Hölderlinjubiläum entwickelten Typographie mit Glyphen. Einen ersten Eindruck über diese räumliche Wirkung kann man sich über ein kurzes Video verschaffen. ${ }^{11}$
Der Lockdown, der bereits nach drei Wochen Laufzeit erfolgt ist, hat diese Ausstellung, die so sehr vom räumlichen Eindruck lebt, besonders stark getroffen. Erst am 8. März konnte man die Ausstellung wiedereröffnen. Bis Ende April soll sie noch präsentiert werden.

Eine virtuelle Ausstellung auf der Grundlage des Tools DDBstudio kann diesen Eindruck natürlich nicht ersetzen. Aber sie kann die Ausstellung ergänzen, vermag punktuelle Schwerpunkte zu setzen und verschiedene Exponate raumunabhängig in den Vordergrund zu rücken. Es lassen sich neue Kombinationen herstellen und sie mittels von Texten und Informationen anreichern. Viele Interessierte, die keine Möglichkeit hatten, die Ausstellung zu besuchen, erhalten so einen Einblick in ausgewählte Themenfelder der Jubiläumsausstellung.

Ideal verbunden werden konnte diese Idee mit einem lohnenswerten Referendarprojekt. Als Mengengerüst haben sich 40 repräsentative Exponate aus der Jubiläumsausstellung angeboten. Als Farbschema wurde ein "natürlich-buntes" Layout ausgewählt, das sich für Themen im Kontext von Tag/Nacht, Herbst, Wald und Natur eignet, zugleich aber auch zur Metaphorik Hölderlins passt.

Jörg Ennen

10) Vgl. Link zur digitalisierten Erstausgabe von Georges „Das Jahr der Seele" (1897): https://digital.staatsbibliothek-berlin.de/werkansicht?P $P N=P P N 82638840 X \& P H Y S I D=P H Y S \_0011$

11) Vgl. https://Www.wlb-stuttgart.de/die-wlb/kulturprogramm/ausstellungen/ausstellung-hoelderlin/ 